\title{
Explosión del feminicidio en El Salvador
}

Elsa Ramos

Investigadora Utec

\section{RESUMEN}

Este artículo de opinión pretende llamar la atención de las y los lectores acerca del grave aumento de los feminicidios en el país.

E tema que se está tratando, a pesar de existir desde tiempos remotos en el país, en razón de que su sociedad se ha desarrollado bajo patrones patriarcales y machistas en donde los diferentes tipos de violencia en contra de la mujer y entre ellos el más grave, el feminicidio han sido visto como "normales", ha permitido que los feminicidios hayan sido poco visibilizados y mucho menos estudiados de forma sistemática. Los esfuerzos que hay a la fecha se han realizado principalmente desde las organizaciones de la sociedad civil que trabajan en defensa de los derechos de la mujer.

\section{Feminicidios}

El fenómeno de los feminicidios se sigue desarrollando en todas las sociedades contemporáneas, o sea, no es un problema que atañe a una o unas sociedades en particular. Lo que no se puede omitir es que, de acuerdo a los diferentes modelos de sociedad que hay actualmente, este fenómeno tiene connotaciones particulares en cada una de estas en específico.
En el caso particular de El Salvador, los crímenes cometidos en contra de las mujeres se suman a la lista de los homicidios. Generalmente el tratamiento que se les da es que son crímenes pasionales. de tal forma que, bajo este cobijo, se le esta disminuyendo al feminicida la responsabilidad y, después, el castigo que se merece.

La categoría feminicidio se empezó a utilizar en la década de los 80 del siglo pasado, pero antes las organizaciones de mujeres y feministas ya habían realizado esfuerzos teóricos, que dieran luz al asesinato de mujeres y las características especiales de género que este tenía. Así, por ejemplo, se utilizaron conceptos como generocidio y ginecidio. Pero no es hasta que sale publicado el libro de Diana Russel y Jill Radford The politics of woman killing, que ellas proponen el termino femicide, el cual es retomado por las organizaciones antes mencionadas, pero no en su traducción literal que significa "asesinato de mujeres". Es entonces que la antropóloga mexicana Marcela Lagarde, quien le pidió la autorización 
a las autoras del libro ya mencionado para retomar el término pero como feminicidio.

De tal manera, Marcela Lagarde nos dice: Plantear que hay feminicidio es plantear algo muy complicado, es darle una explicación política a un problema que ha sido abordado desde un punto de vista policíaco; el viraje que hemos dado es cambiar la tesitura, colocarlo en otro lugar, y es muy importante para poder enfrentar lo que sucede de una mejor manera.

Feminicidio, es una categoría analítica de la teoría política y consiste en enfrentar el problema como parte de la violencia de género contra las mujeres. Este es el primer supuesto epistemológico, teórico y político, y ubicarlo ahí es abordar las causas del feminicidio (Lagarde, 2006).

Más adelante, otras autoras han enriquecido el contenido de esta categoría. Así, por ejemplo, visto desde la perspectiva de los derechos humanos, se nos dice que el feminicidio es la forma extrema de la ausencia de los derechos humanos, es un genocidio de las mujeres, se le puede considerar como un crimen de Estado (Bustos Romero, 2004).

Este artículo ha sido escrito en razón de que en las últimas décadas el aumento de los feminicidios en el país ha adquirido una dimensión nunca imaginada. Lo anterior se ve evidenciado ya que, de acuerdo con un estudio de la Fundación para la Democracia, Seguridad y Paz (Fundemospaz), del año 2007 al 2010, los feminicidios han aumentado en 64\%. De acuerdo con los informes del Observatorio de la violencia de género contra las mujeres, 195 feminicidios se cometieron en el año de 1995; 193 en el 2000; 206 en el 2001; 227 en el 2002; 232 en el 2003; 260 en el 2004; 390 en el 2005; 437 en el año 2006 y para el año 2009, hubo 592 feminicidios, ${ }^{1}$ hasta el mes de octubre del 2010, y de acuerdo con los datos ofre- cidos en el Segundo Informe Nacional sobre la Situación de Violencia contra las Mujeres en El Salvador, 2010 del Isdemu, 417 mujeres fueron asesinadas, pero el dato final de 2010 indica que fueron en total 510 mujeres asesinadas. Es impactante observar como el número de feminicidios aumenta año con año. Se podría decir que, para la extensión territorial del país, este fenómeno en alguna medida se podría considerar ya una pandemia; ninguno de los departamentos que conforman el país se libra de este flagelo.

\section{Feminicidios por departamento en el $2010^{2}$}

\begin{tabular}{|l|c|}
\hline Departamento & Cantidad \\
\hline Ahuachapán & 24 \\
\hline Santa Ana & 46 \\
\hline Sonsonate & 44 \\
\hline La Libertad & 78 \\
\hline San Salvador & 232 \\
\hline Cuscatlán & 15 \\
\hline Chalatenango & 11 \\
\hline La Paz & 27 \\
\hline Cabañas & 7 \\
\hline San Vicente & 8 \\
\hline Usulután & 14 \\
\hline San Miguel & 55 \\
\hline Morazán & 5 \\
\hline La Unión & 13 \\
\hline Total & \\
\hline
\end{tabular}

${ }^{1}$ Estas cifras han sido tomadas de varios números de Las Boletinas y otros informes elaborados por el Observatorio de la violencia de género contra las mujeres de Ormusa. ${ }^{2}$ Cuadro propio, elaborado a partir de los datos proporcionados en Las Boletinas 10 de Ormusa 
Como se observa en la tabla anterior, San Salvador es el departamento con el mayor índice de feminicidios, y en segundo lugar el departamento de la libertad. Como ya se ha afirmado en diferentes investigaciones, en el país hay un déficit de estadísticas que nos indiquen todos los pormenores necesarios sobre el fenómeno de los feminicidios, para poder tomar las decisiones políticas necesarias para encontrarle solución y ponerle fin.

Los datos anteriores tienen el agravante que, de acuerdo a lo informado por el "Instituto de Medicina Legal (IML), en los últimos diez años se ha aumentado el hallazgo de cuerpos de mujeres asesinadas en la vía pública, siendo el grupo etario de 20 a 29 años de edad el de mayor victimización, seguido del grupo de 10 a 19 años. Alrededor del 40\% de ellas con signos de violencia sexual previa y con una creciente evidencia del ensañamiento en sus cuerpos, como decapitaciones, desmembraciones, signos de tortura y de uso de múltiples armas, arma blanca y de fuego" (Juárez, 2010). También en el Informe del Instituto Centro Reina Sofía, del 2010, al elaborarse un ranking por número de víctimas asesinadas por cada millón de mujeres, de los 44 países que se tienen datos sobre feminicidios hasta el año 2006, se tiene que El Salvador se coloca en el primer lugar se ese ranking con 129,43 feminicidios por millón de mujeres. La prevalencia media en Centro y Suramérica ha sido de 45.39 mujeres asesinadas por millón (Sanmartín Espluges, etal., 2010).

Pero no todo son malas noticias. Lo positivo es que, el 25 de noviembre del 2010, la Asamblea Legislativa de El Salvador aprobó la "Ley Especial para una vida libre de violencia para las mujeres", que entrará en vigor a partir de enero del año 2012. En esta ley, en el Artículo 9, se tipifican los tipos de violencia; $y$ en el literal b) se define que la violencia feminicida es la forma extrema de violencia de género contra las mujeres, producto de la violación de sus derechos humanos, en los ámbitos público y privado, conformada por el conjunto de conductas misóginas que conllevan a la impunidad social o del Estado, pudiendo culminar en feminicidio y en otras formas de muerte violenta de mujeres.

Más adelante, en el Título II, relativo a delitos y sanciones del Capítulo I, en el artículo 45 sobre las sanciones al feminicidio y el Artículo 46 sobre feminicidio agravado, se imponen las sanciones correspondientes. A continuación se transcriben textualmente ambos artículos:

\section{Artículo 45.- Feminicidio}

Quien le causare la muerte a una mujer mediando motivos de odio o menosprecio por su condición de mujer, será sancionado con pena de prisión de veinte a treinta y cinco años.

Se considera que existe odio o menosprecio a la condición de mujer cuando ocurra cualquiera de las siguientes circunstancias:

a) Que a la muerte le haya precedido algún incidente de violencia cometido por el autor contra la mujer, independientemente que el hecho haya sido denunciado o no por la víctima.

b) Que el autor se hubiere aprovechado de cualquier condición de riesgo o vulnerabilidad física o psíquica en que se encontraba la mujer víctima.

c) Que el autor se hubiere aprovechado de la superioridad que le generaban las relaciones desiguales de poder basadas en el género.

d) Que previo a la muerte de la mujer el autor hubiere cometido contra ella cualquier conducta calificada como delito contra la libertad sexual. 
e) Muerte precedida por causa de mutilación.

\section{Artículo 46.- Feminicidio Agravado}

El delito de feminicidio será sancionado con pena de treinta a cincuenta años de prisión, en los siguientes casos:

a) Si fuere realizado por funcionario o empleado público o municipal, autoridad pública o agente de autoridad.

b) Si fuere realizado por dos o más personas.

c) Si fuere cometido frente a cualquier familiar de la víctima.

d) Cuando la víctima sea menor de dieciocho años de edad, adulta mayor o sufriere discapacidad física o mental.

e) Si el autor se prevaleciere de la superioridad originada por relaciones de confianza, amistad, doméstica, educativa o de trabajo.

Los dos artículos anteriores se transcriben textualmente, con el objetivo de que sean conocidos, pues en cuanto se publico la noticia acerca de la aprobación de esta ley, de inmediato en un periódico electrónico, muchos hombres reaccionaron de forma violenta y soez en contra de dicha ley, y supongo que sin haberla leído.

A pesar de todas las desconfianzas y temores no sustentados por aquellos sectores de hombres que sienten amenazado su "poderío", la aprobación de esta ley es un paso importante en el avance de la consecución de la igualdad plena de las mujeres salvadoreñas, y de todas aquellas mujeres extranjeras pero que residen en el país, para empezar a vivir sin miedo. Ahora falta esperar que en el proceso de revisión esta ley no vaya a ser mutilada y se rompan todas las buenas expectativas que ella abre para un futuro sin violencia.

\section{Referencias}

- Bustos Romero, Olga. Las mujeres de cd. Juárez: feminicidio y la violencia de género. México, CU, Unam, 2004. Consultado el 4 de enero 2010: catedradh.unesco.unam.mx/generoyequidad/documentos/.../06.ppt-

- Feminicidios en El Salvador subieron 64\% entre 2007 y 2010. Consultado 5 enero 2010:

http://palabrademujer.wordpress.com/2010/11/12/feminicidios-en-el-salvador-subieron-64-entre-2007-y-2010-segun-estudio/

- Instituto Interamericano de Derechos Humanos. Secretaría Técnica. "Situación y análisis del feminicidio en la Región Centroamericana". Consejo Centroamericano de Procuradores de Derechos Humanos, 2006. Consultado el 14 enero 20110: http://www.conadeh.hn/pdf/Femicidio.pdf

- La Boletita 10. Consultada el 13 de enero 2010: http://observatoriodeviolencia.ormusa.org/boletinas/2010_12_BOLETINA_ VG.pdf

- Lagarde y de los Ríos, Marcela. "Feminicidio". Consultado el 17 enero 2010 :

http://www.ciudaddemujeres.com/articulos/Feminicidio

- "Ley especial integral para una vida libre de violencia para las mujeres". Consultada en el 14 enero 2010:

http://www.americalatinagenera.org/es/boletin/documentos/201011-ley-especial-para-vida-libre-violencia.pdf.

- Martínez, Marta. Marcela Lagarde: 'Los feminicidios son la punta del iceberg de todas las formas de violencia cotidiana contra las mujeres'. Consultado en el 20 enero 2010: http://www.adital.com.br/site/noticia.asp?lang=ES\&cod $=47981$

- Sanmartín Espluges, José; Ibarra Marmolejo, Isabel; García Estéves, Yolanda; Martínez Sánchez, Pilar. "III informe Internacional. Violencia contra las mujeres en las relaciones de pareja. Estadísticas y Legislación". España, Centro Reina Sofía; Valencian Internacional University, Instituto Centro Sofía, 2010. Consultado el 21 enero 2010 en: http://www.fundacionluisvives. org/actualidad/noticias/archivo/2010/05/05/iii_informe_internacional_violencia_contra_la_mujer_en_las_relaciones_de pareja_estadisticas_y_legislacion.html. 